\title{
Containing the excitement
}

DOI:

10.1038/nrn2173
Amyloid- $\beta$ (A $\beta)$-rich plaques and tau-containing neurofibrillary tangles are neuropathological hallmarks of Alzheimer's disease (AD). Currently approved drugs for AD target cholinesterase, to reduce acetylcholine degradation, and $N$-methyl-Daspartate (NMDA) receptors, to

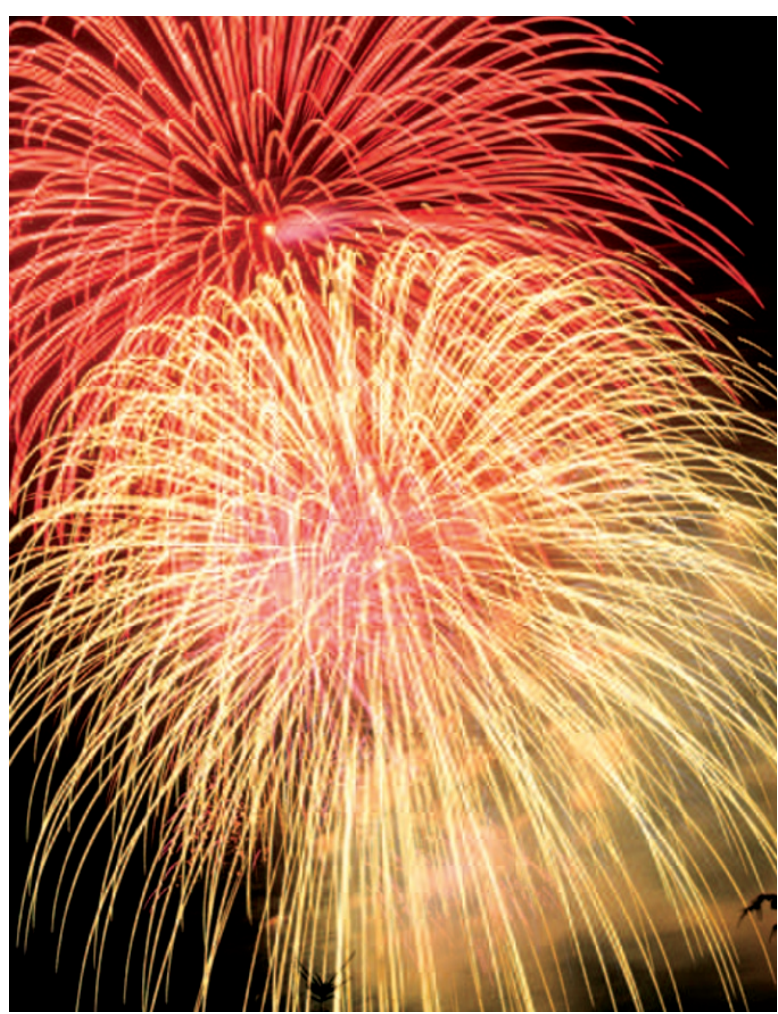

prevent excitotoxicity, but they have limited effects on cognitive impairment. New strategies aiming to block pathogenic $\mathrm{A} \beta$ peptides are now in clinical trials. Mucke and colleagues have opted for an alternative approach, exploring the effects of decreasing endogenous tau levels on A $\beta$-induced cognitive decline. They report that the cognitive deficits in transgenic mouse models of $\mathrm{AD}$ were prevented when the levels of tau were decreased.

The authors crossed mice carrying mutations in the human amyloid precursor protein (hAPP) gene that increase $A \beta$ production with $\mathrm{Tau}^{-1-}$ mice, and examined the learning and memory abilities of their offspring using the Morris water maze. Surprisingly, the deficits observed in the hAPP mice were prevented when tau levels were reduced by $50 \%$. Furthermore, hAPP; $\mathrm{Tau}^{-/-}$mice performed better in spatial learning tasks than hAPP; Tau ${ }^{+/-}$mice, suggesting a gene-dose-dependent effect.

To determine the mechanism through which tau exacerbates the adverse effects of hAPP, Mucke and colleagues examined the levels of hAPP and A $\beta$ in hAPP; $\mathrm{Tau}^{+/-}$and hAPP; $\mathrm{Tau}^{-/-}$mice, and compared them with hAPP mice that expressed normal levels of tau. No changes in the levels of hAPP or levels of A $\beta$ - soluble or aggregated - were observed, suggesting that tau might function to couple $A \beta$ to pathogenesis. However, the decrease in tau did not reduce the amount of neuritic dystrophy surrounding amyloid plaques, or the degree of aberrant axon sprouting in hAPP mice. Instead, the authors found that the reduction of tau prevented neuronal vulnerability to excitotoxicity, a contributing factor to the pathogenesis of $\mathrm{AD}$. In the presence or absence of hAPP/A $\beta$, both $\mathrm{Tau}^{+/-}$and $\mathrm{Tau}^{-/-}$mice were more resistant to excitotoxin-induced seizures than Tau wild-type mice, indicating a novel role for tau in regulating neuronal excitability.

This study not only strengthens the link between aberrant neuronal overexcitation and cognitive impairment, but also highlights the role of tau as a regulator of excitotoxicity, opening new avenues for therapeutic intervention in $\mathrm{AD}$ and other neurodegenerative disorders.

\section{Monica Hoyos Flight}

\section{ORIGINAL RESEARCH PAPER}

Roberson, E. D. et al. Reducing endogenous tau ameliorates amyloid $\beta$-induced deficits in an Alzheimer's disease mouse model. Science $\mathbf{5 8 2 5}$, 750-754 (2007) 